\title{
Cloning of bovine embryos from vitrified donor blastomeres
}

\author{
T. T. Peura ${ }^{1 *}$, M. W. Lane ${ }^{1 *}$, G. Vajta ${ }^{2}$ and A. O. Trounson ${ }^{1}$ \\ ${ }^{1}$ Centre of Early Human Development, Institute of Reproduction and Development, Monash University, Clayton, Victoria, Australia; \\ and ${ }^{2}$ Embryo Technology Center, Danish Institute of Agricultural Sciences, Tjele, Denmark
}

\begin{abstract}
The use of cryopreserved in vitro produced bovine embryos as nuclear transfer donors was assessed. Day 4 or 5 morulae were vitrified and warmed using the open pulled straw method and used as donors for nuclear transfer. Although the proportion of morulae and blastocysts that developed from nuclear transfer embryos derived from day 5 vitrified embryos did not differ from that derived from fresh embryos (16.7 and $24.3 \%$, respectively), development to blastocysts was reduced when vitrified donor cells were used ( 8.3 and $19.1 \%$, respectively). Likewise, development to morulae and blastocysts was not different between nuclear transfer embryos derived from day 4 vitrified embryos allowed to recover for $24 \mathrm{~h}$, and day 5 vitrified embryos allowed to recover for $1-2 \mathrm{~h}$ (27.7 and $15.6 \%$, respectively), but the development to blastocysts was reduced when day 5 vitrified donor cells were used $(23.2$ and $10.0 \%$, respectively). However, in nuclear transfer embryos derived from either day 4 vitrified or day 5 fresh donors, no differences were observed in development rates to morulae and blastocysts ( 34.3 and $36.3 \%$, respectively) or to blastocysts alone ( 20.2 and $18.1 \%$, respectively). Nor were there differences in the development rates of fresh or day 4 or day 5 vitrified in vitro produced (non-nuclear transfer) embryos $(47.9,51.0$ and $35.5 \%$ developing to blastocysts at day 7 , respectively). In vitro produced embryos and nuclear transfer embryos derived from day 4 vitrified or day 5 fresh donors were transferred to recipients at morula or blastocyst stage at day 6 or 7 . The pregnancy rates were similar in both groups of nuclear transfer embryos, but higher in the control group consisting of in vitro produced embryos (47, 42 and $67 \%$, respectively). In conclusion, if vitrified donor embryos are allowed to recover for $24 \mathrm{~h}$ after warming, their use in nuclear transfer results in similar efficiencies to those achieved with fresh embryos.
\end{abstract}

\section{Introduction}

Fundamental biological questions of development and differentiation can be resolved by nuclear transfer, where the nucleus of an oocyte or zygote is replaced with the nucleus of a developmentally more advanced cell. This method has also been used to produce offspring in livestock species (Willadsen, 1986; Prather et al., 1987). In the majority of bovine nuclear transfer experiments, the donor embryos are used fresh without cryopreservation. Early studies in bovine embryo cloning used mainly in vivo- derived embryos, flushed from superovulated and inseminated donor animals on the day of nuclear transfer (Willadsen et al., 1991). Improved in vitro techniques have since facilitated the production of donor embryos for nuclear transfer purposes (Zakhartchenko et al., 1996). However, there is a need for a long-term storage of in vivo and in vitro produced embryos. The successful production of large numbers of identical

*Present address: South Australian Research and Development Institute, Turrerfield Research Centre, Rosedale, SA 5350, Australia. Received 17 August 1998. offspring from a single embryo relies on recycling nuclear transfer embryos as donor cells for subsequent rounds of nuclear transfer. However, this approach is limited by several factors. The inability to control the progression of the recycling process once it has commenced and the lack of abattoir-derived oocytes on weekends limits the number of recycling rounds that can be performed. In addition, full use of a large number of clones derived from one original donor embryo becomes increasingly difficult as the number of recycling rounds increases. The ability to cryopreserve nuclear transfer donor embryos successfully would remove these limitations in recycling and enable the recycling to be fully controlled, thus improving the overall efficiency of cloning techniques.

Few studies have compared the developmental capacity of nuclear transfer embryos derived from cryopreserved or non-cryopreserved embryos. Westhusin et al. (1991) and Yang et al. (1993) compared fresh and frozen-thawed in vivoderived bovine embryos as donors in nuclear transfer, and observed no differences in fusion or embryonic development rates. However, Heyman et al. (1994) observed a significant reduction in embryonic development after using vitrified- 
warmed in vivo-derived bovine embryos as compared with non-vitrified controls. Likewise, Ushijima et al. (1996) reported reduced development rates when in vitro produced bovine donor embryos had been delipated and vitrified at the onecell stage and used as cell donors for nuclear transfer at the 8-16-cell stage.

A novel vitrification technique called the open pulled straw technique (OPS) has been developed and successfully applied to embryos of various developmental stages and species (Vajta et al., 1998). Preliminary reports from our laboratory (Peura et al., 1998a) as well as from the laboratory where this technique was developed (Vajta et al., 1997a) indicate that this technique can be applied to nuclear transfer donor embryos. In the present study, the effects of the developmental stage of donor embryos at the time of vitrification and the recovery time after warming on the nuclear transfer outcome were evaluated, to establish optimal procedures for using vitrified donor embryos in bovine embryo cloning.

\section{Materials and Methods}

\section{Production of embryos and oocytes}

Bovine oocytes and embryos were produced in vitro as described by Peura et al. (1998b). Briefly, immature oocytes were aspirated from abattoir-derived ovaries and matured in groups of 50 in $800 \mu \mathrm{l}$ TCM-199 (Gibco BRL, Grand Island, NY) containing 10\% fetal calf serum (FCS; Gibco) and $0.01 \mathrm{iu}$ $\mathrm{ml}^{-1}$ bovine LH and bovine FSH (NOBL Laboratories, Sioux Center, IA) at $39^{\circ} \mathrm{C}$ in an atmosphere of $5 \% \mathrm{CO}_{2}$ in air. Donor embryos were produced from oocytes fertilized after 22-24 h maturation in groups of ten in $50 \mu \mathrm{l}$ fertilization drops with frozen-thawed Percoll (Sigma Chemical Co., St Louis, MO) separated bull spermatozoa at a concentration of $2 \times 10^{6}$ spermatozoa $\mathrm{ml}^{-1}$, at $39^{\circ} \mathrm{C}$ in an atmosphere of $5 \% \mathrm{CO}_{2}$ in air. Twenty-four hours after fertilization, cumulus cells were removed by vortexing and the putative zygotes were cultured for 3 days at $39^{\circ} \mathrm{C}$ in an atmosphere of $5 \% \mathrm{CO}_{2}, 7 \%$ $\mathrm{O}_{2}$ and $88 \% \mathrm{~N}_{2}$ in $500 \mu \mathrm{l}$ modified synthetic oviductal fluid (mSOF) (Edwards et al., 1997) containing Eagle's nonessential amino acids (ICN Biomedicals Inc., Aurora, $\mathrm{OH}$ ) and $8 \mathrm{mg} \mathrm{BSA} \mathrm{ml}^{-1}$ (Sigma) in four-well culture dishes (Nunc, Roskilde). The embryos were then cultured in mSOF containing Eagle's essential and non-essential amino acids (ICN) and $8 \mathrm{mg} \mathrm{BSA} \mathrm{ml}^{-1}$ for a further 3 days. Handling of immature oocytes was done at room temperature, whereas all subsequent stages of oocyte and embryo handling were performed on warm stages (LEC Instruments, Scoresby, Vic) at $38^{\circ} \mathrm{C}$.

\section{Embryo vitrification and warming}

In vitro produced embryos were vitrified either at day 4 or day 5 (day 0 =day of fertilization). In either case, only embryos at the expected stage of cleavage and development were vitrified according to the method of Vajta et al. (1997a). Briefly, embryos were first washed in a bench medium consisting of TCM-199, 25 mmol Hepes $\mathrm{l}^{-1}$ (Sigma) and 20\%
FCS, followed by $3 \mathrm{~min}$ incubation in the bench medium containing 7.5\% (v/v) dimethylsulfoxide (DMSO) (Sigma) and $7.5 \%(\mathrm{v} / \mathrm{v})$ ethylene glycol (Sigma). The embryos were then incubated for $25 \mathrm{~s}$ in the bench medium containing 0.6 mol sucrose $\mathrm{l}^{-1}$ (Sigma), $16.5 \%(\mathrm{v} / \mathrm{v})$ DMSO and $16.5 \%(\mathrm{v} / \mathrm{v})$ ethylene glycol, during which time they were loaded into thin pulled straws by capillary action, in approximately $2 \mu \mathrm{l}$ medium, followed by immediate plunging into liquid nitrogen. The straws were prepared from $0.25 \mathrm{ml}$ insemination straws (IMV, L'Aigle) by heating them above a coiled heating plate and pulling gently to achieve long, narrow straws. These were then cut with a razor blade to yield straws approximately $9 \mathrm{~cm}$ long and $0.8 \mathrm{~mm}$ outer diameter at the narrow end. For storage, straws were stored in loosely closed $15 \mathrm{ml}$ conical tubes in liquid nitrogen.

Embryos were removed one straw at a time from liquid nitrogen and warmed in air for $2-3 \mathrm{~s}$ before submerging the narrow end of the straw into the first well of a four-well Nunc plate containing $1.2 \mathrm{ml}$ bench medium with $0.3 \mathrm{~mol}$ sucrose $\mathbf{1}^{-1}$. The other end of the straw was covered by a fingertip, causing the embryos to expel from the straw into the medium, where they were incubated for $5 \mathrm{~min}$. The embryos were then transferred to the second well containing bench medium with $0.15 \mathrm{~mol}$ sucrose $1^{-1}$ for another $5 \mathrm{~min}$ incubation, and then incubated twice, $5 \mathrm{~min}$ each, in the third and fourth well in the bench medium without sucrose. Embryos were then cultured in mSOF culture medium containing Eagle's essential and non-essential amino acids and $8 \mathrm{mg} \mathrm{BSA} \mathrm{ml}^{-1}$.

\section{Nuclear transfer and embryo culture}

Recipient oocytes were enucleated as described by Peura et al. (1998b). Briefly, cumulus cells were removed by vortexing and mature oocytes were selected according to the presence of an extruded first polar body. After agglutinating polar bodies to oocytes with phytohaemagglutinin (ICN) incubation and removing zonae pellucidae with Pronase (Sigma), oocytes were bisected unequally with a metal blade by free hand. The smaller portions of the oocytes (approximately $40 \%$ of the total oocyte volume) still attached to the polar body were discarded and the remaining, chromatin-free cytoplasts were used for nuclear transfer. This method routinely results in $>95 \%$ enucleation efficiency, as demonstrated by chromatin staining with the fluorochrome Hoechst 33342 (Sigma) and examination under ultraviolet illumination. Cytoplasts were subsequently activated in $38 \mathrm{mmol} \mathrm{Ca}^{2+}$-ionophore $\mathrm{l}^{-1}$ (Sigma) in proteinfree TCM-199 for 10 min followed by a $4 \mathrm{~h}$ incubation in 2 mmol 6-dimethylaminopurine (6-DMAP) $1^{-1}$ (Sigma) in TCM-199 plus 10\% FCS, after which they were used for cell fusions.

Donor embryo disaggregations and nuclear transfers were performed as described by Peura et al. (1998b). Briefly, after removal of zona pellucida with pronase, donor embryos were disaggregated into individual blastomeres in cytochalasin B-supplemented medium. Cytoplasts and blastomeres were fused in mannitol fusion medium in an electrofusion chamber containing parallel wires with a 
diameter of $0.5 \mathrm{~mm}$ and a separation of $0.5 \mathrm{~mm}$. For each nuclear transfer embryo, two cytoplasts and one blastomere were aligned with an AC pulse of 8-10 V and $500-600 \mathrm{kHz}$ for 5-10 s, then fused with a single DC pulse of $100 \mu \mathrm{s}$ and an amplitude of $600 \mathrm{~V} \mathrm{~cm}^{-1}$. Reconstituted embryos were cultured individually in $20 \mu \mathrm{l}$ drops. For the first 3 days, the embryos were cultured in mSOF supplemented with Eagle's non-essential amino acids and $20 \mathrm{mg} \mathrm{BSA} \mathrm{ml}^{-1}$ and, after that, in mSOF supplemented with Eagle's essential and nonessential amino acids and $20 \mathrm{mg} \mathrm{BSA} \mathrm{ml}^{-1}$. Embryo cleavage and developmental stage were recorded 3,6 and 7 days after fusion. The number of blastocyst cells was determined by placing embryos in an ethanol fixative containing $25 \mathrm{mg}$ Hoechst $33342 \mathrm{ml}^{-1}$ at $4^{\circ} \mathrm{C}$ in the dark overnight. Embryos were then mounted in glycerol (Sigma) on a microscope slide, pressed down with a coverslip and the fluorescent nuclei were counted under ultraviolet light using an excitation filter of $340-380 \mathrm{~nm}$.

\section{Experiment 1}

Fresh and vitrified then warmed day 5 embryos were assessed as nuclear transfer donors. Briefly, some morulae at day 5 of culture were vitrified, warmed immediately and returned to the culture for another $3 \mathrm{~h}$, while the rest of the embryos were left in culture. Embryos from both groups were used subsequently as donors for nuclear transfer. Recipient cytoplasts were produced earlier the same day and used immediately after the end of DMAP incubation. Reconstituted embryos from both groups were cultured under identical conditions until day 7 , and fusion, cleavage and development rates were recorded.

\section{Experiment 2}

Embryos were vitrified and warmed either at day 4 or day 5 , allowed to recover for 24 or $1-2 \mathrm{~h}$, respectively, after warming and assessed as nuclear transfer donors. Briefly, precompaction stage morulae at day 4 of culture were selected and divided into two groups. Embryos in one group were vitrified, immediately warmed, changed into fresh medium and cultured for an additional $24 \mathrm{~h}$. Embryos in the other group were changed into fresh medium, cultured for an additional $24 \mathrm{~h}$, after which the morphologically normal embryos (average $90 \%$ of embryos left to culture) were vitrified, immediately warmed and returned to culture for 1-2 h. Embryos from both groups were used as nuclear donors for nuclear transfer, using the recipient cytoplasts prepared earlier on that day. Reconstituted embryos were cultured under identical conditions until day 7. Fusion, cleavage and development rates to morulae and blastocysts, and total cell numbers of blastocysts were recorded.

\section{Experiment 3}

Embryo development after vitrification and warming either at day 4 or day 5 was examined to assess whether developmental stage affected the survival of vitrified embryos. Precompaction stage morulae at day 4 of culture were selected and divided into three groups. Embryos in the control group were changed into fresh medium and cultured until day 7. Embryos in the second group were vitrified, immediately warmed, changed into fresh medium and cultured until day 7. Embryos in the third group were changed into fresh medium, cultured for an additional $24 \mathrm{~h}$, after which the morphologically normal embryos were vitrified, immediately warmed and returned to culture until day 7. Development rates to morulae and blastocysts for all groups as well as blastocyst total cell numbers were recorded.

\section{Experiment 4}

Nuclear transfer embryos were produced using the best protocol as demonstrated in Expts 1 and 2 and transferred to recipients to evaluate the viability of nuclear transfer embryos derived from vitrified donors. As controls, in vitro produced embryos and nuclear transfer embryos derived from fresh donor embryos were also transferred. At day 4 after fertilization, a group of in vitro produced precompaction stage morulae were vitrified and immediately warmed, whereas the rest of the embryos were left intact. After a further $24 \mathrm{~h}$ in culture, embryos of both groups were used as donors for nuclear transfer. The resulting reconstituted embryos were cultured for 6 or 7 days and transferred to recipients. In vitro control embryos were produced separately, cultured for 7 days and transferred to the same group of recipients. Embryos were loaded in each insemination straw in Hepes-buffered mSOF medium containing $16 \mathrm{mg} \mathrm{BSA} \mathrm{ml}^{-1}$ and transferred to recipients within $4 \mathrm{~h}$ of loading, during which time straws were kept at $37^{\circ} \mathrm{C}$. Fifteen recipients received nuclear transfer embryos derived from fresh donors, fourteen receiving three and one receiving four embryos. Nuclear transfer embryos derived from vitrified donors were transferred to a total of 12 recipients, 11 receiving three and one receiving four embryos. Control embryos were transferred to a total of six recipients, each receiving two embryos. Recipients for embryo transfer were synchronized with an intravaginal progesterone-releasing device (CIDR ${ }^{\oplus}$, InterAg, Hamilton) inserted for 8 days, with a prostaglandin i.m. injection (Estruplan ${ }^{\circledast}$, Parnell, Sydney) given $24 \mathrm{~h}$ before CIDR removal. Non-surgical embryo transfers into the uterine horn ipsilateral to the corpus luteum were performed 6-7 days after standing oestrus was first observed. Pregnancy was determined by ultrasonography 40 days after embryo transfer. The number of fetuses in each recipient, as identified by ultrasonographic examination, was recorded.

\section{Statistical analyses}

Fusion rates were determined as the proportion of successfully fused, reconstituted embryos from the number of attempted fusions. All subsequent cleavage and embryo development rates were calculated from the total number 


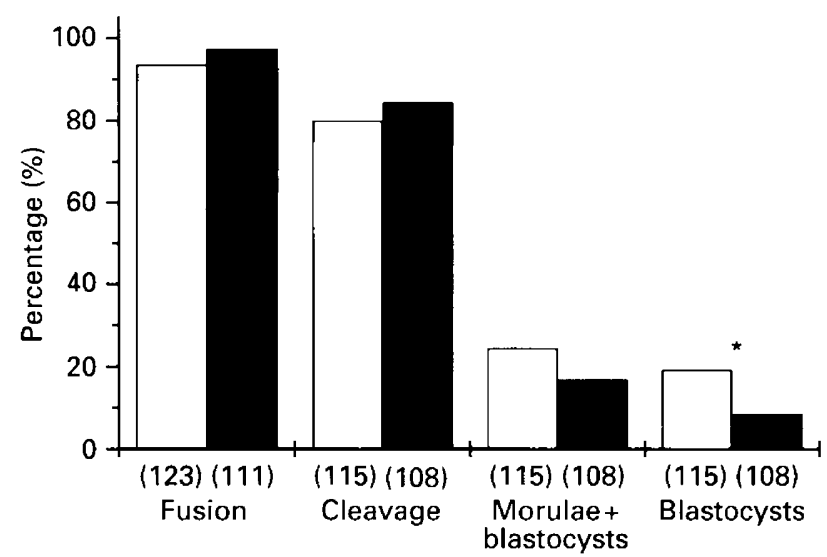

Fig. 1. Fusion and development rates of bovine nuclear transfer embryos derived from in vitro produced day 5 fresh $(\square)$ or vitrified $(\square)$ donor embryos. *Difference between columns significant $(P<0.05)$. Numbers of embryos fused or cultured are in parentheses.

of successfully fused embryos. Fusion, cleavage and development rates between the experimental groups in all four experiments were compared by unpaired $t$ tests (Expts 1,2 and 4 ) or by the analysis of variance (ANOVA; Expt 3) after arcsin transformation of the original percentage data. Differences in embryonic cell numbers were compared without the data transformation. In both cases, the statistical package Graphpad Instat ${ }^{\mathrm{TM}}$ (GraphPad Software) was used.

\section{Results}

\section{Experiment 1}

A total of 14 fresh and 11 vitrified day 5 donor embryos were used for nuclear transfer in five replicate experiments. Mean cell numbers obtained from the embryos after disaggregation were $27.6 \pm 2.5$ from fresh and $34.0 \pm 3.3$ from vitrified (mean \pm SEM). Fusion and cleavage rates did not differ significantly from each other between the two groups. Although there were no differences in the development rates to morulae and blastocysts at day 7 ( 24.3 and $16.7 \%$ for fresh and vitrified donors, respectively) (Fig. 1), development to blastocysts was significantly better $(P=0.0221)$ for nuclear transfer embryos derived from fresh donors as opposed to vitrified donors (19.1 versus $8.3 \%$, respectively).

\section{Experiment 2}

A total of 54 donor embryos were vitrified and warmed in five replicate experiments. Some embryos $(<10 \%$ of total) were lost during the warming procedures, and further losses occurred as some embryos did not recover from the procedures but degenerated soon after warming $(20-30 \%$ of total). After disaggregation, since embryos yielding $<20$ blastomeres were rejected (as is routinely done with fresh donors), only 24 embryos were eventually used for nuclear

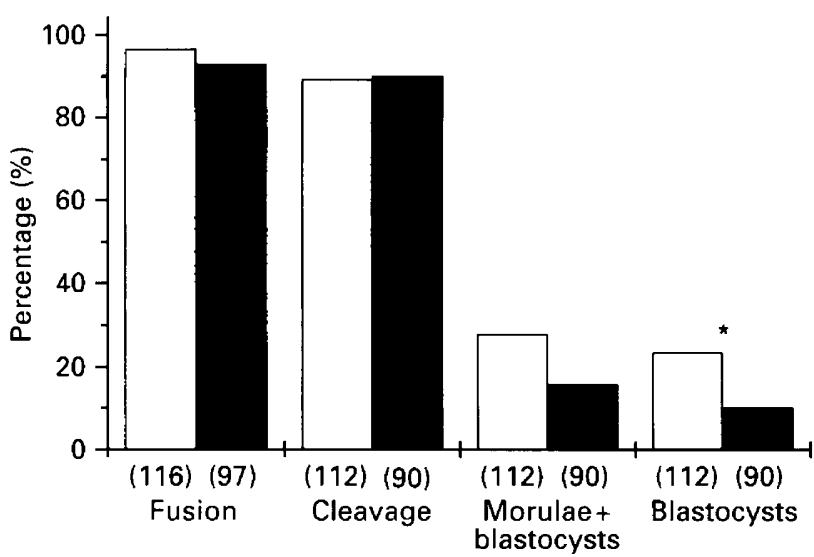

Fig. 2. Fusion and development rates of bovine nuclear transfer embryos derived from in vitro produced day $4(\square)$ or day 5 ( $\square$ ) vitrified donor embryos, allowed to recover after vitrification for 24 or $1 \mathrm{~h}$, respectively. ${ }^{*}$ Difference between columns significant $(P<0.05)$. Numbers of embryos fused or cultured are in parentheses.

transfer after disaggregation. There were no differences in the numbers of blastomeres obtained from embryos vitrified at day 4 compared with those vitrified at day $5(25.3 \pm 2.6$ and $27.1 \pm 2.8$, respectively). Again, fusion and cleavage rates did not differ significantly between the two groups (Fig. 2). Differences were neither observed in development rates to morulae or blastocysts at day $7(27.7$ and $15.6 \%$, with day 4 and day 5 vitrified donors, respectively) (Fig. 2). However, development to blastocysts was significantly better $(P=0.0290)$ in nuclear transfer embryos derived from day 4 vitrified donors than it was in day 5 vitrified donors (23.2 versus $10.0 \%$, respectively). Total cell numbers of nuclear transfer blastocysts were not significantly different between the two groups $(P=0.2944)$. A mean $( \pm$ SEM $)$ of $87.2 \pm 7.9$ and $97.5 \pm 5.6$ cells were identified in blastocysts derived from day $4(n=18)$ and day $5(n=8)$ vitrified donors, respectively.

\section{Experiment 3}

More than 1500 oocytes were fertilized and cultured in five separate replicates to produce 623 precompaction stage day 4 embryos. The overall cleavage rate was $73.1 \%$ $(1150 / 1573)$. Only grade 1 quality embryos on each day (day 4 and day 5) were vitrified, resulting in only $56 \%$ of the embryos selected initially being vitrified at day 5 . The recovery rate of embryos after warming was $84 \%$. Owing to this loss in recovery, subsequent embryonic development rates are expressed for all groups as actual development rates $\left(\%_{\text {cult }}\right)$, on the basis of the development of embryos cultured from each group. Development is also expressed as corrected development rate $\left(\%_{\text {corr }}\right)$, on the basis of the number of embryos initially selected for each group. Although day 5-vitrified embryos had the highest actual development rates, compared with day 4-vitrified and control embryos (75.0, 59.1 and $63.3 \%$ to morulae and blastocysts, and $59.1,51.0$ and $47.9 \%$ to blastocysts, 
Table 1. Development rates of in vitro produced bovine embryos after vitrification-warming at day 4 or 5 after insemination (control: day 4 non-vitrified embryos)

\begin{tabular}{|c|c|c|c|c|c|c|c|c|c|}
\hline \multirow[b]{2}{*}{ Group } & \multicolumn{3}{|c|}{ Number of embryos } & \multicolumn{3}{|c|}{ Morulae + blastocysts } & \multicolumn{3}{|c|}{ Blastocysts } \\
\hline & Selected ${ }^{a}$ & Vitrified & Cultured & $n$ & $\%_{\text {cult }}$ & $\%_{\text {corr }}$ & $n$ & $\%_{\text {cult }}$ & $\%_{\text {corr }}$ \\
\hline Control & 165 & n.a. & 165 & 104 & $63.3^{\mathrm{b}}$ & $63.3^{b}$ & 79 & $47.9^{b}$ & $47.9^{b}$ \\
\hline Vitrified at day 5 & 178 & 107 & 88 & 66 & $75.0^{\mathrm{b}}$ & $45.1^{\mathrm{c}}$ & 52 & $59.1^{b}$ & $35.5^{b}$ \\
\hline
\end{tabular}

${ }^{a}$ All embryos for the experimental groups were selected at day 4 after fertilization.

$\%_{\text {cul' }}$ percentage of the actual number of cultured embryos developing to morulae or blastocysts; $\%{ }_{\text {corr }}$ corrected percentage of embryos selected to the experimental group developing to morulae or blastocysts.

b.cValues with different superscripts within a column differ significantly $(P<0.05)$

respectively), they also had the lowest corrected development rate $(45.1,59.1$ and $63.3 \%$ to morulae and blastocysts, and $35.5,51.0$ and $47.9 \%$ to blastocysts, respectively). No differences among groups were observed in actual development rates or, in development to blastocysts, in corrected development rates (Table 1.). However, the difference in corrected development rates to morulae and blastocysts was significantly lower in day 5vitrified donors $(P=0.0236)$. Total cell numbers (mean \pm SEM) of the blastocysts were $95.1 \pm 6.1$ in the control group ( $n=$ 29), $99.2 \pm 4.7(n=45)$ in the day 4-vitrified group and $98.7 \pm$ $9.1(n=24)$ in the day 5-vitrified group. These cell numbers were not significantly different among groups.

\section{Experiment 4}

In vitro development rates of nuclear transfer embryos derived from day 4 -vitrified and day 5 fresh donor embryos are shown (Fig. 3). As in Expts 1 and 2, there were no differences in blastomere numbers obtained after the disaggregation of embryos vitrified at day 4 and cultured overnight when compared with day 5 fresh embryos (35.8 \pm 3.0 and $27.1 \pm 3.0$, respectively). No statistically significant differences were observed in fusion or cleavage rates or in development rates to morulae and blastocysts (34.3 and $36.3 \%$ for vitrified and fresh donors, respectively) or to blastocysts (20.2 and $18.1 \%$ for vitrified and fresh donors, respectively) at day 6 . The development rates were only recorded up to day 6 as, in two out of three replicates, the embryos were transferred into recipients at day 6 . The pregnancy rates achieved are shown (Table 2).

Overall, from the pooled results of Expts 1,2 and 4, these numbers of blastomeres (mean \pm SEM) were obtained from disaggregated donor embryos: $32.1 \pm 2.1$ from fresh, $26.4 \pm$ 2.0 from day 4-vitrified and $30.7 \pm 2.3$ from day 5-vitrified donors. These numbers were not significantly different among the three groups.

\section{Discussion}

These experiments indicate that, when appropriate vitrification and warming procedures are used, there are no significant differences in the developmental potential of

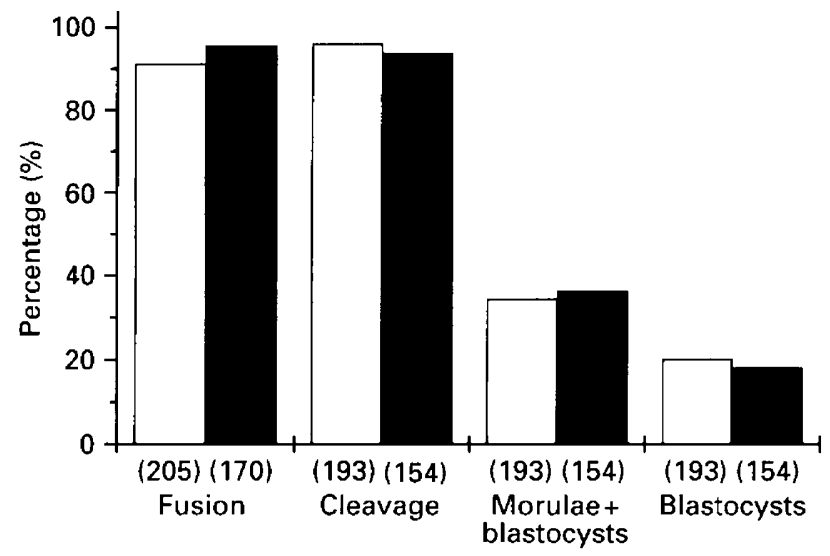

Fig. 3. Fusion and development rates of bovine nuclear transfer embryos derived from in vitro produced donor embryos, vitrified at day 4 and allowed to recover after vitrification for $24 \mathrm{~h}(\square)$, or fresh embryos used at day $5(\boldsymbol{\square})$. Differences between columns were not significant $(P<0.05)$. Numbers of embryos fused or cultured are in parentheses.

bovine nuclear transfer embryos derived from in vitro produced fresh or cryopreserved donor embryos. Although development rate to blastocysts was decreased when vitrified donor embryos were used for nuclear transfer shortly after warming, this deleterious effect could be overcome by allowing a longer recovery period before use. The advantages of this approach are twofold. First, the embryos subjected to extensive cryodamage can be easily identified and discarded at the end of prolonged culture period according to their morphology. Second, embryos only slightly affected by the vitrification procedure will benefit from the prolonged recovery period. Vajta et al. (1997b) observed serious subcellular injuries in vitrified bovine blastocysts when viewed by electron microscopy. In addition to severe overall degeneration of some cells, ultrastructural damage was observed in mitochondria and in intracellular contacts, but this damage was restored progressively over $24 \mathrm{~h}$. However, in the case of nuclear transfer, recovery of intracellular organelles and intracellular contacts is not essential because the recipient ooplasm provides all the cytoplasmic components needed. Therefore, the improved development may reflect the identification and avoidance of donor cells containing seriously cryodamaged nuclei. 
Table 2. Day 40 pregnancy rates of in vitro produced and nuclear transfer bovine embryos derived from vitrified or fresh donor embryos

\begin{tabular}{|c|c|c|c|}
\hline Group & $\begin{array}{c}\text { Number of transferred embryos / } \\
\text { number of recipients }\end{array}$ & Number of fetuses ${ }^{a}$ & $\begin{array}{c}\text { Number of recipients } \\
\text { pregnant }\end{array}$ \\
\hline IVP control embryos & $12 / 6$ & $5(42 \%)$ & $4(67 \%)$ \\
\hline NT-embryos from fresh donors & $46 / 15$ & $14(30 \%)$ & $7(47 \%)$ \\
\hline NT-embryos from vitrified donors & $38 / 12$ & $7(18 \%)$ & $5(42 \%)$ \\
\hline
\end{tabular}

at least this many fetuses observed by ultrasonography.

IVP, in vitro produced; NT, nuclear transfer.

The cryopreservation method may play a role in comparison of fresh and cryopreserved morulae as donors in nuclear transfer. Westhusin et al. (1991) and Yang et al. (1993) found no detrimental effect of cryopreservation of day 5-6 in vivo embryos for nuclear transfer outcomes, whereas Heyman et al. (1994) found reduced development after cryopreservation of day 5.5 in vivo embryos. The difference between these results could be explained by the different cryopreserving techniques used. Traditional freezing by slow-cooling methods was used by Westhusin et al. (1991) and Yang et al. (1993) and vitrification was used by Heyman et al. (1994). Slow-cooling techniques for freezing late morula and blastocyst stage bovine embryos have been modified over the years, whereas vitrification techniques are still relatively new in embryo cryopreservation. However, slowcooling techniques have not been successful when used to cryopreserve early preimplantation stage embryos (Pollard and Leibo, 1994). The high lipid content and high volume to surface ratio of bovine early preimplantation stage embryos renders them susceptible to cryoinjury induced by ice crystal formation and osmotic damage, both of which occur during freezing by slow-cooling. Besides the developmental stage, the origin of the embryo (in vivo or in vitro) affects its ability to survive freezing (Leibo et al., 1996). The embryos used for cryopreservation in the current study were day 4 and 5 in vitro produced embryos at early pre-compaction and compacted morula stages. Previous investigations revealed that these embryos do not survive freezing by traditional slow-cooling techniques (T. Peura and I. Lewis, unpublished). Delval et al. (1998) used a different vitrification method for in vitro produced day 6 bovine embryos, which were subsequently used as donors for nuclear transfer. Although no comparison was made with non-vitrified donor embryos, the reported development rates after nuclear transfer were quite good ( $20.5 \%$ blastocysts from reconstituted embryos). The novel vitrification method used throughout this study has been used successfully for cryopreservation of embryos previously deemed to be extremely sensitive to cryoinjury, such as pig embryos and bovine oocytes (Vajta $e t$ al., 1998). The major advantage of the OPS is the very high cooling and warming rate achieved because of the novel design of the micro-straws $\left(20000^{\circ} \mathrm{C} \mathrm{min}^{-1}\right.$ as compared with $2500^{\circ} \mathrm{C}$ $\mathrm{min}^{-1}$ with traditional $0.25 \mathrm{ml}$ mini-straws).

The origin of the donor embryo may also be an important factor in defining the developmental potential after nuclear transfer. However, the comparison of nuclear transfer embryos derived from in vivo and in vitro donor embryos reveals some discrepancies among different studies. Superior development of in vivo-derived nuclear transfer embryos has been reported by Yang et al. (1993) and Clement-Sengewald et al. (1992). Heyman et al. (1994) and Zakhartchenko et al. (1996) found no differences in the developmental potential of in vivo compared with in vitro derived nuclear transfer embryos. In the present study, all donor embryos were produced in vitro, yielding rates of in vitro development comparable with those of other studies. Development rates to morulae and blastocysts in the present study varied from 24 to $36 \%$ in fresh day 5 donors, from 28 to $34 \%$ in day 4 vitrified donors, and from 16 to $17 \%$ in vitrified day 5 donors. The present results support those of Booth et al. (1998), who observed no differences between in vitro developmental potential of nuclear transfer embryos derived from fresh or vitrified donors ( 40.1 versus $33.7 \%$ blastocysts, respectively).

Vajta et al. (1998) found impaired embryo development when embryos were vitrified at day 1 and day 2, but no differences from day 3 to day 7 . The results of the present study confirm this. No differences were found in the developmental potential of in vitro produced embryos after vitrification at either day 4 or day 5 after fertilization. The number of blastomeres obtained from donor embryos was not significantly affected by vitrification, even though day 4vitrified embryo groups yielded a slightly smaller number of blastomeres than day 5-vitrified embryos and day 5 fresh control embryos (means of 26.4, 30.7 and 32.1 blastomeres, respectively). Although this difference was not statistically significant, it is possible that the trend observed results from the interruption and subsequent retardation of development by vitrification. Yang et al. (1993) reported reduced blastomere recovery rates from frozen embryos when compared with fresh embryos (from $90 \%$ recovery rate to $60 \%$ ), but a similar effect was not observed in the present study. Hardly any cell lysis was observed in vitrified embryos at disaggregation.

The percentage of recipients pregnant at day 40 did not differ between nuclear transfer embryos derived from fresh or vitrified donor embryos but, in both groups, it was lower than in vitro control embryos (47, 42 and $67 \%$, respectively) (Table 2). Initially, quite high fetal survival rates were obtained from control and fresh donor groups as compared with vitrified donor group (at least 42,30 and 18\% of transferred embryos developing into fetuses, respectively). Ten of sixteen pregnant recipients were diagnosed as carrying multiple fetuses, either two or three per recipient, but some of these multiple pregnancies in the fresh donor 
group were subsequently lost between days 40 and 110 . This is not surprising, as low pregnancy rates and high pregnancy losses are common with nuclear transfer embryos (Yang et al., 1993). In addition, increased fetal losses have been reported to result from uterine overcrowding when more than one fetus is present in the same uterine horn (Hanrahan 1983), as was the case in the present study. In view of the present results, the number of embryos transferred to recipients may need to be reduced to improve the chances of the development of early fetuses to live offspring.

This research was supported by the Dairy Research and Development Corporation, Meat Research Corporation and Genetics Australia Co-op. Soc. Ltd. The authors also thank K. Boekel for her skillful assistance in embryo manipulations, and I. Lewis, J. Owens, M. Ryan and Genetics Australia for providing recipients and their management.

\section{References}

Booth PJ, Vajta G, Holm P, Jacobsen H, Greve T and Callesen H (1998) Open pulled straw (OPS) vitrification of both cytoplasts and day 3 embryos donors in bovine nuclear transfer Theriogenology 49384

Clement-Sengewald A, Palma GA, Berg U and Brem G (1992) Comparison between in vitro produced and in vivo flushed donor embryos for cloning experiments in cattle Theriogenology 37196

Delval A, Ectors FJ, Beckers J-F, Ectors F and de Loos F (1998) Vitrification of day 6 in vitro produced bovine embryos as donors for cloning by nuclear transfer Theriogenology 49165

Edwards LJ, Batt PA, Gandolfi F and Gardner DK (1997) Modifications made to culture medium by bovine oviduct epithelial cells: changes to carbohydrates stimulate bovine embryo development Molecular Reproduction and Development 46 146-154

Hanrahan JP (1983) Inter-ovarian distribution of twin ovulations and embryo survival in the bovine Theriogenology 20 3-11

Heyman Y, Chesne P, Lebourhis D, Peynot N and Renard JP (1994) Developmental ability of bovine embryos after nuclear transfer based on the nuclear source: in vivo versus in vitro. Theriogenology 42 695-702
Leibo SP, Martino A, Kobayashi S and Pollard JW (1996) Stage-dependent sensitivity of oocytes and embryos to low temperatures Animal Reproduction Science 42 45-53

Peura TT, Lane MW, Lewis IM and Trounson AO (1998a) The use of vitrified multigenerational cloned cattle embryos as donors in nuclear transfer Theriogenology 49326

Peura TT, Lewis IM and Trounson, AO (1998b) The effect of recipient oocyte volume on nuclear transfer in cattle Molecular Reproduction and Development 50 185-191

Pollard J and Leibo SP (1994) Chilling sensitivity of mammalian embryos Theriogenology 41 101-106

Prather RS, Barnes FL, Sims MM, Robl JM, Eyestone WH and First NL (1987) Nuclear transplantation in bovine embryo: assessment of donor nuclei and recipient oocyte Biology of Reproduction 37 859-866

Ushijima H, Yamakawa $H$ and Nagashima $H$ (1996) Nucleus transfer of bovine IVM/IVF embryos: cryopreserved delipated 8-16 cell stage blastomeres as donor nuclei Proceedings of the International Congress on Animal Reproduction, 30 June-4 July 1996, Sydney, Australia, P21-22

Vajta G, Booth PJ, Holm P, Greve T and Callesen H (1997a) The use of vitrified day 3 embryos as donors in bovine nuclear transfer Cryo-Letters 18 355-358

Vajta G, Hyttel P and Callesen H (1997b) Morphological changes of in vitro produced bovine blastocysts after vitrification, in-straw direct hydration and culture Molecular Reproduction and Development 48 9-17

Vajta G, Kuwayama M, Holm P, Booth PJ, Jacobsen H, Greve T and Callesen $\mathbf{H}$ (1998) A new way to avoid cryoinjuries of mammalian ova and embryos: the OPS-vitrification Molecular Reproduction and Development 51 53-58

Westhusin M, Pryor J and Bondioli K (1991) Nuclear transfer in the bovine embryo: a comparison of 5-day, 6-day, frozen-thawed and nuclear transfer donor embryos Molecular Reproduction and Development 28 119-123

Willadsen SM (1986) Nuclear transplantation in sheep embryos Nature 320 63-65

Willadsen SM, Janzen RE, McAlister RJ, Shea BF, Hamilton G and McDermand D (1991) The viability of late morulae and blastocysts produced by nuclear transplantation in cattle Theriogenology 35 161-170

Yang X, Jiang S, Farrell P, Foote RH and McGrath AB (1993) Nuclear transfer in cattle: effect of nuclear donor cells, cytoplast age, co-culture and embryo transfer Molecular Reproduction and Development 35 29-36

Zakhartchenko V, Reichenbach H-D, Riedl J, Palma GA, Wolf E and Brem G (1996) Nuclear transfer in cattle using in vivo-derived versus in vitro produced donor embryos: effect of developmental stage Molecular Reproduction and Development 44 493-498 